\title{
Isolated tricuspid valve infective endocarditis with multiple septic pulmonary emboli in a patient with atopic dermatitis
}

Nobuki Shioya ${ }^{1}$, Nozomu Inoue ${ }^{1}$, Erina Miyano ${ }^{2}$, Miyabi Nakayama ${ }^{1}$, Yuki Tsukamoto ${ }^{1}$, Koji Hazama ${ }^{3}$, and Yasuo Shichiohe ${ }^{4}$

${ }^{1}$ National Hospital Organization Hokkaido Medical Center

${ }^{2}$ National Hospital Organization Hokkaido Medical Center

${ }^{3} \mathrm{NHO}$ Hokkaido Medical Center

${ }^{4}$ National Hospital Organization Hokkaido Medical Center

May 11, 2020

\begin{abstract}
Multiple lung nodules in atopic dermatitis patients may reflect infective endocarditis. Our case underlines the importance of potentially severe infections due to staphylococci associated with atopic dermatitis.
\end{abstract}

\section{The title of the paper}

Isolated tricuspid valve infective endocarditis with multiple septic pulmonary emboli in a patient with atopic dermatitis

\section{The full names of the authors}

Nobuki Shioya, Nozomu Inoue, Erina Miyano, Miyabi Nakayama, Yuki Tsukamoto, Koji Hazama, and Yasuo Shichinohe

The addresses of the institutions

Critical Care and Emergency Center

National Hospital Organization Hokkaido Medical Center

1-1, 5-7 Yamanote, Nishi-Ku, Sapporo, Japan 063-0005

Contact address

Nobuki Shioya

Critical care and emergency center, Hokkaido Medical Center, National Hospital Organization

1-1, 5-7 Yamanote, Nishi-Ku, Sapporo, Japan 063-0005

Tel: +81-(11)-611-8111, Fax: +81-(11)-611-5820

E-mail:funamushi002764@gmail.com

\section{KEYWORDS}

atopic dermatitis, multiple septic pulmonary embolism, isolated tricuspid valve infective endocarditis

KEY CLINICAL MESSAGE 
Atopic dermatitis may cause infective endocarditis associated with staphylococci. Generally, Right-sided infective endocarditis responds well to medication, but our patient had multiple organ failure and required long-term ICU management.

A 51-year-old man with severe atopic dermatitis (AD) visited our hospital with high fever and general malaise. On physical examination, his body temperature was $39.8^{\circ} \mathrm{C}$, heart rate was $129 / \mathrm{min}$, blood pressure was $115 / 71 \mathrm{mmHg}$, respiratory rate was 25 breaths/min, and oxygen saturation was $97 \%$ under room air. Laboratory tests showed the following results: white cell count $17,600 / \mu \mathrm{L}, \mathrm{C}$-reactive protein $32.4 \mathrm{mg} / \mathrm{dL}$, and procalcitonin $17.3 \mathrm{ng} / \mathrm{mL}$. Chest computed tomography showed multiple cavities in bilateral lungs, consistent with septic emboli (Figure 1). Transthoracic echocardiography revealed a vegetation on the tricuspid valve (Figure 2). Blood cultures revealed methicillin-sensitive Staphylococcus aureus .

It is generally reported that right-sided Infective endocarditis (IE) displays a good response to medical therapy. However, our patient initially suffered from multiple organ failure, so we performed long-term ventilation management and blood purification.

IE due to $S$. aureus occasionally occur in patients with $\mathrm{AD}$, because AD lesions are often colonized by $S$. aureus $^{1}$, and the biofilm is almost ubiquitous in AD lesion skin. The perivalvular abscess and the valve perforation as $S$. aureus -linked IE manifestation of tissue destruction were associated with high mortality 2 .

\section{CONFLICT OF INTEREST}

The authors report no conflict of interest.

\section{REFERENCES}

1. Benenson S, Zimhony O, Dahan D et al . Atopic dermatitis - a risk factor for invasive Staphyolococcus aureus infections: two cases and review. Am J Med. 2005; 118 (9): 1048-51.

2. Damlin A, Westling K, Maret E et al. Associations between echocardiographic manifestations and bacterial species in patients with infective endocarditis: a cohort study. BMC Infect Dis. 2019; 19 (1):1052.

\section{FIGURE LEGENDS}

Figure 1: Axial computed tomography section in lung on admission shows multiple nodules (arrows) and cavitary infiltrates (A-D), feeding vessel sign (arrowhead) (C), and bilateral pleural effusion in bilateral lungs.

Figure 2: Transthoracic echocardiogram shows a huge vegetation (arrow heads) of $3 \mathrm{~cm}$ or more attached to the tricuspid valve extending to the pulmonary artery (A, B).

\section{AUTHOR CONTRIBUTIONS}

NS: involved in conception and design, manuscript preparation.

NI: made diagnosis, obtained and edited images, and prepared.

EM and MN: obtained images and reviewed the manuscript.

YT, KH and YS : reviewed the manuscript. 


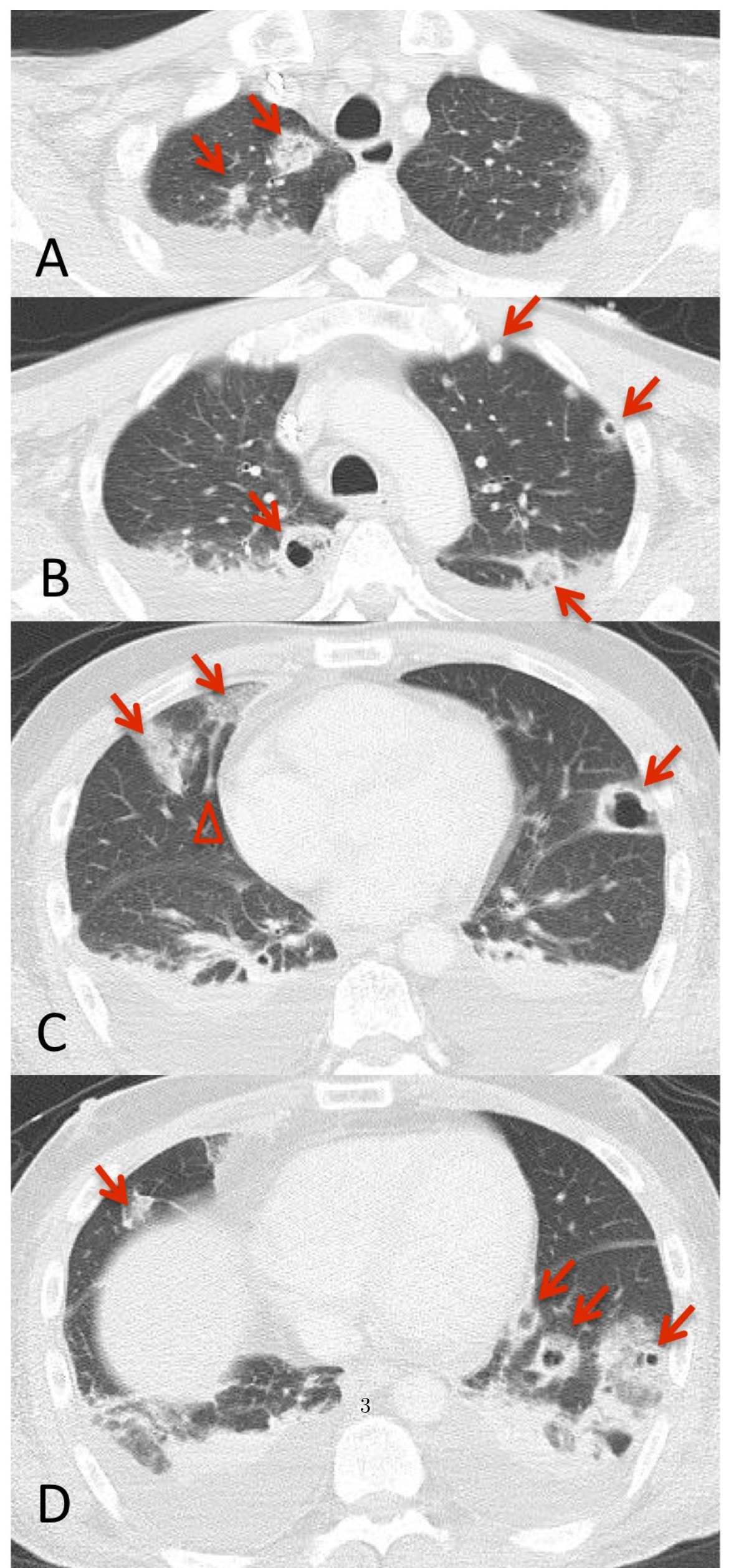




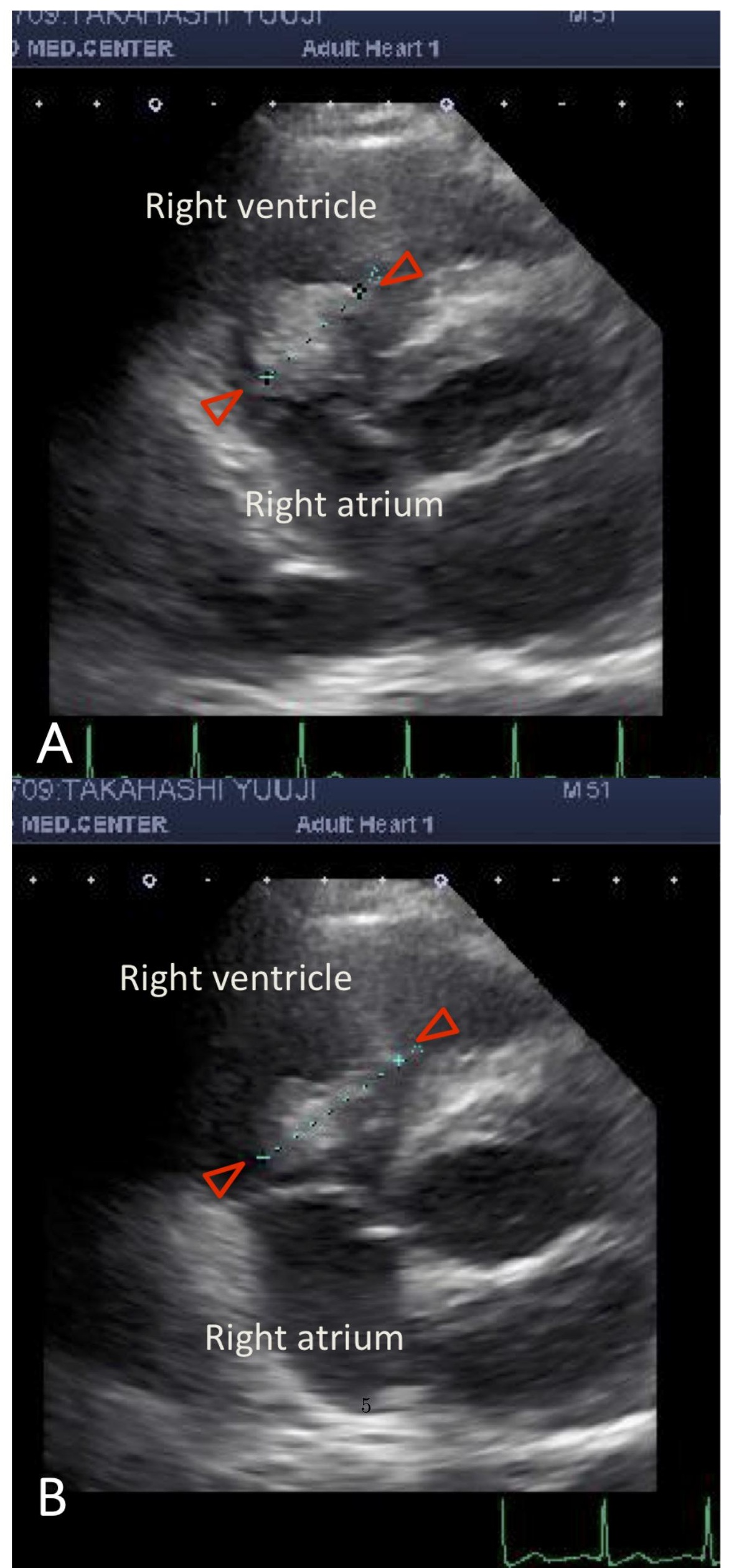

